journal club

\title{
KVT durch „Dr. Internet“ bessert Insomnie
}

Fragestellung: Kann ein vollautomatisches, internetbasiertes Programm zur Durchführung einer kognitiven Verhaltenstherapie der Insomnie (KVT-I) eine signifikante Verbesserung des Schweregrads einer Insomnie erreichen und ist ein solches Programm einer reinen psychoedukativen Wissensvermittlung in einer heterogenen Patientengruppe überlegen?

Hintergrund: Die medikamentösen Strategien zur Therapie einer chronischen Insomnie sind aufgrund des Nebenwirkungsprofils vor allem in der Langzeitbehandlung limitiert. Die in vielen Studien gezeigte gute Wirksamkeit und teilweise Überlegenheit der KVT-I hat dazu geführt, dass diese als Goldstandard angesehen wird. Leider führen der Mangel an ausreichend ausgebildeten Therapeuten und der notwendige Personalaufwand oft dazu, dass eine notwendige Behandlung nicht erfolgen kann. Daher konzentriert sich ein wichtiger Teil der Insomnieforschung auf neue Techniken zur Vermittlung von KVT-I.

Patienten und Methodik: In einer einfach verblindeten, randomisierten klinischen Studie wurden in einem Parallelgruppendesign 303 Patienten im Alter zwischen 21 und 65 Jahren mit chronischer Insomnie entwe-

Ritterband LM, Thorndike FP Ingersoll KS et al. Effect of a web-based cognitive behavior therapy for insomnia intervention with 1-year follow-up: A randomized clinical trial. JAMA Psychiatry 2017; 74: 68-75 der einer internetbasierten KVT-I oder standardisierten Psychoedukation über neun Wochen zugeordnet. Alle eingeschlossenen Patienten zeigten relevante Veränderungen des Schlafs in Selbstbeurteilungsfragebögen.
Ausgeschlossen wurden Patienten mit organischen Schlafund Schlaf-Wach-Rhythmusstörungen, Schwangere und solche mit abgeschlossener oder gerade stattfindender anderweitiger psychotherapeutischer Behandlung. Patienten mit komorbiden psychischen Erkrankungen wurden hingegen eingeschlossen, wenn aufgrund Erkrankungsschwere, -stabilität und -behandlung eine Teilnahme möglich war. Die Auswertung erfolgte mit Schlaftagebüchern (zwei Wochen) und mit einem etablierten Fragebogen zum Insomnieschweregrad (ISI) prä- und postinterventionell sowie als Follow-up nach sechs und zwölf Monaten. Etwa 250 Patienten absolvierten die Studie vollständig.

Ergebnisse: Als Haupteffekte wurden die Veränderung in ISI, nächtlichen Wachzeiten (Schlaftagebuch, WASO) und Einschlaflatenz (Schlaftagebuch, SOL) über die verschiedenen Messzeitpunkte analysiert. Es zeigte sich eine signifikante Interaktion Gruppe $\times$ Zeit für ISI und WASO über alle Zeitpunkte, die in weiteren Untersuchungen als Überlegenheit der Verbesserung nach KVT-I gegenüber Psychoedukation konkretisiert wurde. Innerhalb der einzelnen Gruppen zeigten sich sowohl für KVT-I als auch für Psychoedukation eine signifikante Verbesserung, die auch nach einem Jahr bestand. Die Effektstärke war dabei deutlich höher für die KVT-I. Diese Effekte wurden durch eine psychiatrische Komorbidität nicht beeinflusst.

Schlussfolgerungen: Die Autoren interpretieren ihre Ergebnisse als Beweis, dass eine automatisierte, internetbasierte KVT-I eine adäquate Behandlung der Insomnie ermöglicht und einer reinen internetbasierten Psychoedukation überlegen ist.

\section{- Kommentar von Lukas Frase und Dieter Riemann, Freiburg i. Br.}

\section{Automatisierte Psychotherapie - eine interessante Perspektive}

Die Arbeit liefert eine fachlich gut konzipierte und statistisch einwandfreie Grundlage für eine weitere klinische Anwendung internetbasierter Psychotherapien (Evidenzstufe II). Bei der Behandlung der Insomnie ist eine KVT die nach Studienlage beste Therapieoption, jedoch oft nicht verfügbar. Ein automatisiertes, internetbasiertes Programm, das interaktiv genug ist, um ähnliche Effekte wie eine klassische Psychotherapie zu liefern, könnte daher die Behandlung sehr vieler Patienten verbessern. Ein direkter Wirksamkeitsvergleich zwischen „echtem“ Therapeuten und PC-Programm ist in der aktuellen Studie zwar nicht gegeben, die Ergebnisse sind aber gut und mit der Studienlage vergleichbar. Als Einschränkung wird berichtet, dass die höhere Teilnahme von weißen Frauen, die in ihrem Alltag häufig das Internet nutzen, eine Generalisierung auf andere Patientenkollektive erschwert. Zudem fand keine Objektivierung der Beschwerden, etwa durch eine Polysomnografie statt. Vergleichbare Studien könnten sich auch bei anderen Krankheitsbildern anbieten, die primär psychotherapeutisch behandelbar sind, beispielsweise Angsterkrankungen und Zwangsstörungen. Hier liegen erste Studien vor [1], eine vollautomatisierte Behandlungsoption ist aber nicht etabliert [2].
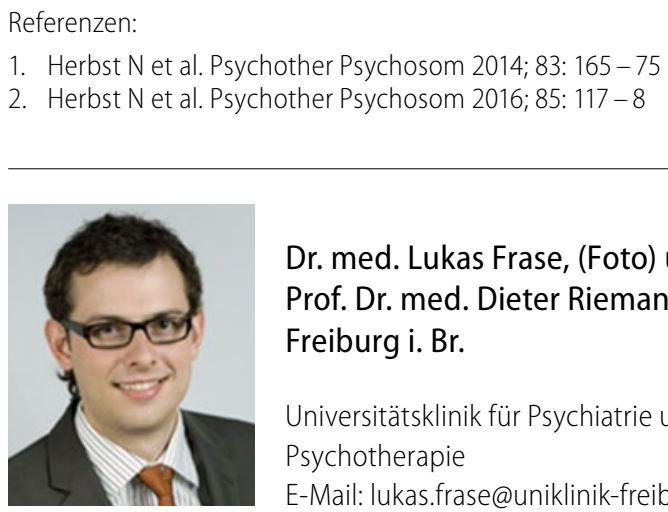

Dr. med. Lukas Frase, (Foto) und Prof. Dr. med. Dieter Riemann, Freiburg i. Br.

Universitätsklinik für Psychiatrie und Psychotherapie

E-Mail: lukas.frase@uniklinik-freiburg.de 